\title{
Effect of early school timings on mood and performance of students
}

\section{Ruchi Singh*; Renuka Sharma*; J.C. Suri"; Raj Kapoor"; Shobha Das*}

* Department of Physiology, \# Department of Pulmonary, Critical Care \& Sleep Medicine, Vardhman Mahavir Medical College \& Safdarjung Hospital, New Delhi

Indian J Sleep Med 2010; 5.3, 88-94

\begin{abstract}
Introduction: Early school timings along with delayed sleep phase syndrome causes adolescents to lose sleep during the school week. Studies have attributed insufficient sleep as one of the factors contributing to poor school performance of students. Sleep deprivation can impair memory and concentration, making it difficult for students to learn. Irritability, lack of self confidence and mood swings are common in teenagers, and sleep deprivation makes matters worse. This study compared the academic performance, sleep habits and mood changes in Indian student attending the same school in two different shifts.

Methods: A questionnaire-based cross sectional study was conducted among 834 students of $\mathrm{VI}$ to IX standard attending different shifts [Shift I (7:00 am to 1:10 pm) and Shift II (1 1:30 am to 6:00pm)].The questionnaires evaluated the students for their sleep pattern, duration, napping, quality of sleep and mood. Daytime sleepiness was scored using the Epworth Sleepiness Scale. Performance was calculated by their percentages in respective subjects and a total score was taken for their overall performance during the academic session.

Result: Baseline characteristics of both the groups were comparable with mean age of $12.9 \pm 1.33$ (shift I) \& 12.9 \pm 1.21 (shift II). Shift II students had significantly greater total sleep time with only $19 \%$ of them reporting sleep deprivation (total sleep time $<8 \mathrm{hrs}$ ) as compared to $58 \%$ of shift I. Shift I students reported daytime tiredness in $8.4 \%$, napping in $51.7 \%$ and sleep awakenings at night in $58.1 \%$ which was significantly more as compared to Shift II students. Sleepiness in prelunch classes $(P=0.001)$ as well as consumption of caffeine/tea $(P=0.0001)$ was also more in Shift I. Overall performance of shift II students was better than shift I, with students getting significantly better grades in English $(P=0.001)$ and Mathematics $(P=0.003)$.

Conclusion: This study demonstrated that early school timings contribute significantly to sleep deprivation among adolescents, leading to daytime sleepiness and poorer academic performance. Apart from delayed sleep phase syndrome, other social engagements may be the contributory factors for the late bed timings leading to sleep deprivation in morning shift students. Further studies, involving students of different socioeconomic group of different cities, are recommended to find the various factors and the extent to which each of them are responsible for change in sleep habits affecting mood and academic performance.
\end{abstract}

Keywords: school timings, adolescents, delayed sleep phase syndrome, performance, mood.

\section{Address for correspondence}

Prof (Dr) J C Suri

Consultant \& Head - Department of Pulmonary

Critical Care \& Sleep Medicine, Safdarjang Hospital

New Delhi. Email: jcsuri@rediffmail.com 


\section{Introduction}

S leep patterns among adolescents have recently been observed to be significantly deviated from the established norms provoking great concern amongst educational and health professionals. Habitual early risers in childhood develop an altered schedule of staying up late at night and rising much later in the day ${ }^{(1,2)}$. The shift in the sleep patterns is a result of several factors like changes in the intrinsic period of the circadian clock, behavioral and social factors as well as delayed circadian sleep phase syndrome(DSPS) making early bed times difficult to be achieved. DSPS occurs with a frequency of $0.17 \%$ in the general population and $7 \%$ in the adolescents ${ }^{(17)}$. The higher prevalence of delayed sleep phase in adolescents is likely the result of a combination of biological (lengthening of the circadian period) ${ }^{(3)}$ and behavioral (social schedules) factors $^{(4)}$.

Adequate sleep is defined as 8-9 hrs of regular, night sleep. However, early school start timings combined with sleeping late lead to significant sleep loss during the week days ${ }^{(5,6)}$. Decreased sleep duration in adolescents has been attributed as one of the factors for poor school performance, as it negatively affects social, behavior and quality of life. Irritability, lack of self confidence and mood swings are often common in teenagers, and sleep deprivation makes it worse. These dangerous repercussions of sleep deprivation are more widely prevalent than realized.

However, data on these side effects and their relation to adolescent sleep remains scanty due to the limited studies conducted so ${ }^{(7,8,9,10)}$. The objective of the present study was to understand the effect of school timing on sleep hygiene of the students as well as its impact on their mood and academic performance.

\section{Materials and Methods}

A cross sectional study was conducted among the students of a school in Delhi between grade sixth to ninth after obtaining a requisite permission from the school administration. The students were enrolled after a written, informed consent from their parents. The school had two shifts. Shift I (Morning shift 7:00 am to 1:10 pm) and Shift II (Evening shift 11:30 am to 6:00 pm). Consent form for the parents was distributed to all the students of grade sixth to ninth of both the shifts. Of 1000 forms distributed, consent was received from 900 parents. Further data was collected only from students who consented to the study.

The rationale of the study was explained to the participants prior to the administration of questionnaires. Students were administered validated questionnaires for analyzing their sleep habits, sleep duration, period of nap, quality of sleep, sleep awakenings separately for weekdays $\&$ weekends $s^{(11)}$. Questionnaire was in easy, understandable language and individual items were explained to the participants. They were clearly instructed not to fill the responses where they were unsure. Daytime sleepiness was scored using Epworth Sleepiness Scale $(\text { ESS })^{(12)}$ where values of ESS score $>10$ indicated excessive sleepiness. Anxiety and depression among the students was also assessed using standardized questions. Regarding substance abuse, the students were asked for consumption of caffeine, sleeping pills, alcohol and cigarettes. Performance was calculated based on their marks in respective subjects and total score in all the subjects was taken for their overall performance during the academic session. Ethical clearance for the study was obtained from the Institutional Ethics committee of Vardhman Mahavir Medical College and Sadarjang Hospital, New Delhi.

\section{Statistical Methods}

Data analysis was done using SPSS version 16.0 (SPSS Inc, Chicago, IL, USA). The normality of data was tested using Shapiro Wilk test. Unpaired t-test and chi-square test were used for testing the differences in the prevalence's by characteristics. Correlation analysis was performed using Spearman's correlation coefficient. A two-tailed $(\alpha=2)$, probability ( $\mathrm{p}$ ) value less than 0.05 $(\mathrm{p}<0.05)$ was considered to be statistically significant for all statistical tests applied.

\section{Result}

Eight hundred and thirty four forms complete in all respects, were included in the study. Shift I students comprised of 501 students and 333 students were of Shift II. Students of both groups were comparable in their baseline characteristics but showed marked differences in sleep routines and daytime sleepiness (Table-1).

\section{Sleep routines of the students}

On comparing the sleep routines of the two groups (Table-2) it was found that a significantly higher number $(74.5 \%)$ of Shift I students went to bed before $10 \mathrm{pm}$ on weekdays as compared to $62.5 \%$ of Shift II students. Total sleep duration of Shift I students was significantly less on

Indian Journal of Sleep Medicine (IJSM), Vol. 5, No. 3, 2010 
week days ( $\mathrm{p}=0.0001$ ) leading to sleep deprivation (total sleep $<8 \mathrm{hrs}$ ) in a $58 \%$ of students. No difference was observed in the sleep routines of the two groups on weekends (Table-2). It was also seen that $31 \%$ of students in each shift slept when they felt drowsy, but a significant $(\mathrm{p}=0.0001)$ number of Shift I students had to be compulsive woken from sleep.

On comparing the relative frequency of time to bed, it was observed that a majority of students of both shifts

Table 1: Baseline characters of the Subjects

\begin{tabular}{|l|c|c|c|}
\hline VARIABLES & $\begin{array}{c}\text { Shift I } \\
(\mathbf{n}=501)\end{array}$ & $\begin{array}{c}\text { Shift II } \\
(\mathbf{n}=333)\end{array}$ & $\begin{array}{c}\text { "p" } \\
\text { Value }\end{array}$ \\
\hline Age & $12.9 \pm 1.33$ & $12.9 \pm 1.21$ & 0.344 \\
\hline Height & $150.2 \pm 13.85$ & $149.3 \pm 12.98$ & 0.345 \\
\hline Weight & $42.09 \pm 9.73$ & $40.9 \pm 9.21$ & 0.089 \\
\hline $\begin{array}{l}\text { Time to bed } \\
\text { on week days }\end{array}$ & $22.17 \pm 0.72$ & $22.38 \pm 0.89$ & $.000^{* *}$ \\
\hline Total sleep duration & $7.0 \pm 0.97$ & $8.10 \pm 1.08$ & $.000^{* *}$ \\
\hline ESS & $6.94 \pm 3.98$ & $6.46 \pm 3.62$ & .070 \\
\hline
\end{tabular}

Table 2: Comparison of sleep routines of the students of two shifts

\begin{tabular}{|l|l|c|c|c|}
\hline \multicolumn{2}{|c|}{ VARIABLES I } & Shift II & "p" Value & \\
\hline Bedtime before 10pm & Weekdays & $74.5 \%$ & $62.5 \%$ & $0.0001^{* *}$ \\
\cline { 2 - 5 } & Weekend & $47.1 \%$ & $45.8 \%$ & 0.708 \\
\hline $\begin{array}{l}\text { Total sleep time } \\
\text { (Sleepe } \geq 8 \text { hrs) }\end{array}$ & Weekdays & $41.9 \%$ & $81.1 \%$ & $0.0001^{* *}$ \\
\cline { 2 - 6 } & Weekend & $81.6 \%$ & $81.7 \%$ & 0.987 \\
\hline $\begin{array}{l}\text { Sleep Deprivation } \\
\text { (Sleep time <8 hrs) }\end{array}$ & $58 \%$ & $19 \%$ & $0.0001^{* *}$ \\
\hline Going to bed when sleepy & $31 \%$ & $31 \%$ & 0.998 \\
\hline $\begin{array}{l}\text { Wake up due to compulsion } \\
\text { (parents or alarm) }\end{array}$ & $92.6 \%$ & $83.2 \%$ & $0.0001^{* *}$ \\
\hline
\end{tabular}

preferred to sleep between 10-11pm (Graph-1). A significant number of Shift I students slept between 9pm$10 \mathrm{pm}$ compared to Shift II students who slept mostly between 10-11pm.

While comparing the total duration of sleep on weekdays, it was seen that a significantly greater number of Shift I students slept for periods of 5-7 hrs as compared to Shift-II students who had an average sleep durations of 8-10 hrs (Graph-2).

Indian Journal of Sleep Medicine (IJSM), Vol. 5, No. 3, 2010

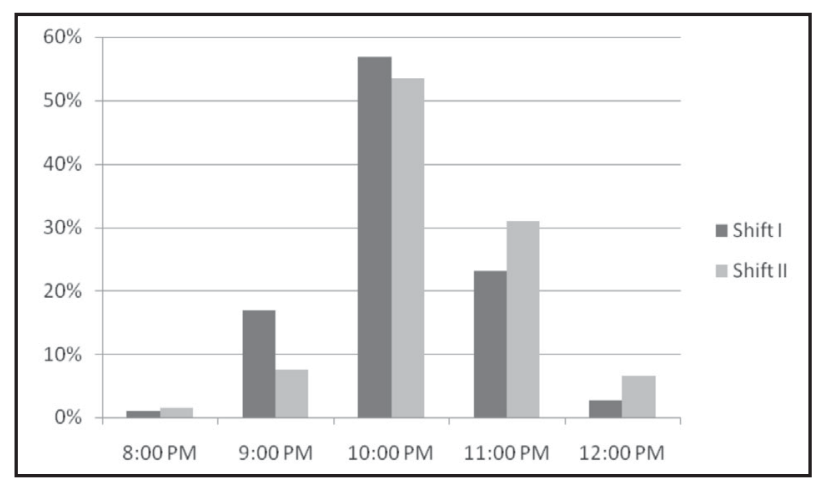

Graph-1: Comparison of relative frequency of students for time to bed

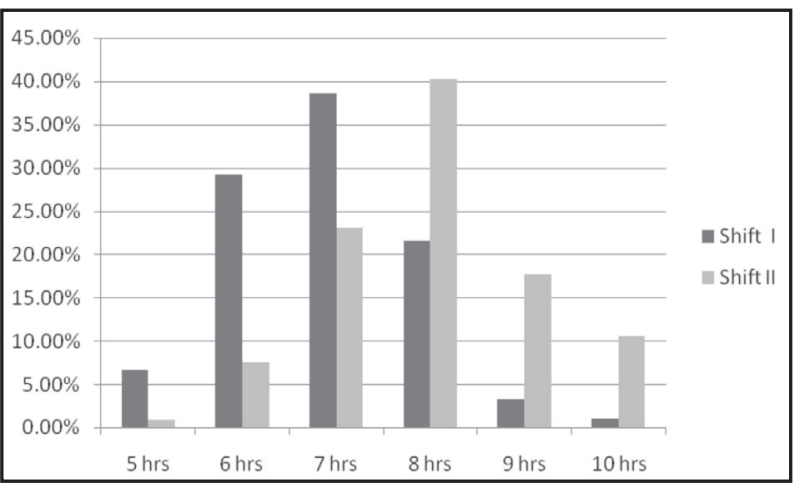

Graph 2: Comparison of total sleep duration on weekdays of the two shifts

\section{Effects of sleep loss}

Sleep loss among Shift I students was associated with significantly higher incidence of tiredness (8.4\%), napping $(51.7 \%)$ and increased consumption of tea/coffee (4.2\%). These students also had a significantly greater prevalence of reporting late to schools (3.8\%) and drowsiness in the morning classes. Shift I children also experienced difficulty in waking up in the morning $(58.1 \%)$ and displayed higher daytime sleepiness. Sleep disruption like frequent awakenings were also significantly more $(\mathrm{p}=0.002)$ in morning shift students (Table-3) .

\section{Performance of the students of two shifts}

Overall performance was observed to be better in Shift II students. On comparing the performance in individual subjects, it was found that Shift II students performed better in all subjects and had significantly higher scores in English $(\mathrm{p}=0.001)$ and Mathematics $(\mathrm{p}=0.003)$ (Table - 4) 
Table 3: Effects of sleep loss

\begin{tabular}{|l|c|c|c|}
\hline VARIABLES & $\begin{array}{c}\text { Shift I } \\
(\mathrm{N}=501)\end{array}$ & $\begin{array}{c}\text { Shift II } \\
(\mathrm{N}=333)\end{array}$ & $\begin{array}{c}\text { 'p' } \\
\text { Value }\end{array}$ \\
\hline Tired during day & $8.4 \%$ & $4.8 \%$ & $0.003^{* *}$ \\
\hline Napping & $51.7 \%$ & $36.6 \%$ & $0.0001^{* *}$ \\
\hline ESS > 10 & $20 \%$ & $15.9 \%$ & 0.133 \\
\hline Tea/Coffee( $\geq 3$ times/day) & $4.2 \%$ & $2.4 \%$ & $0.0001^{* *}$ \\
\hline Difficulty in waking & $58.1 \%$ & $51.7 \%$ & 0.067 \\
\hline Frequent late comers (to school) & $3.8 \%$ & $1 \%$ & $0.0001^{* *}$ \\
\hline Sleepiness in pre-lunch classes & $10.6 \%$ & $7.2 \%$ & $0.001^{* *}$ \\
\hline Sleep in post-lunch classes & $13.2 \%$ & $13.8 \%$ & 0.339 \\
\hline Sleep awakenings & $12.6 \%$ & $11.7 \%$ & $0.002^{* *}$ \\
\hline
\end{tabular}

Table: 4 Performance of students of the two shifts in different subjects

\begin{tabular}{|l|c|c|c|}
\hline SUBJECTS & $\begin{array}{c}\text { Shift I } \\
(\mathrm{N}=333)\end{array}$ & $\begin{array}{c}\text { Shift II } \\
(\mathrm{N}=501)\end{array}$ & $\begin{array}{c}\text { "p" } \\
\text { Value }\end{array}$ \\
\hline English & $61.27 \pm 18.07$ & $65.45 \pm 18.04$ & $0.001^{* *}$ \\
\hline Science & $61.01 \pm 18.96$ & $63.07 \pm 18.29$ & 0.119 \\
\hline Social Studies & $63.33 \pm 20.35$ & $64.24 \pm 18.21$ & 0.506 \\
\hline Mathematics & $59.73 \pm 18.73$ & $63.53 \pm 17.1$ & $0.003^{* *}$ \\
\hline Overall & $372.74 \pm 98.39$ & $378.73 \pm 90.45$ & 0.374 \\
\hline
\end{tabular}

\section{Effects on Mood}

No significant differences were demonstrated in the mood of students of the two shifts, but the level of anxiety and hopelessness was seen to be more in morning batch students (Table-5).

Table 5: Mood changes in the two groups

\begin{tabular}{|l|c|c|c|}
\hline VARIABLES & $\begin{array}{c}\text { Shift I } \\
(\mathrm{N}=501)\end{array}$ & $\begin{array}{c}\text { Shift II } \\
(\mathrm{N}=333)\end{array}$ & $\begin{array}{c}\text { 'p' } \\
\text { Value }\end{array}$ \\
\hline Depression & $19.8 \%$ & $20.7 \%$ & 0.735 \\
\hline Anxiety & $16 \%$ & $12.9 \%$ & 0.223 \\
\hline Hopelessness & $9 \%$ & $7.8 \%$ & 0.552 \\
\hline
\end{tabular}

\section{Discussion}

This study showed that students of Shift I reported higher prevalence of sleepiness and tiredness during the daytime, often reaching late to school. Shift I students had significantly early bed timings but their total sleep time was significantly less leading to sleep deprivation (total sleep $<8 \mathrm{hrs}$ ) in $58 \%$ of students. Consequently, reporting a significant incidence of napping and increased consumption of stimulants to overcome sleepiness.
Impaired memory and concentration, due to sleepiness appears to be responsible for the poorer performance of Shift I students as compared to Shift II studensts. At least 8-9 hours of night time sleep has been suggested for attaining optimal daytime alertness ${ }^{(13,14,15)}$. Lower sleep durations leads to sleep debt causing a vicious cycle of increased daytime sleepiness and reduced performance.

\section{Sleep routines of the students}

The present study demonstrates a significant difference in the sleep routines in the students of the two shifts on schooldays as compared to weekends or holidays. On weekdays, the percentage of students of Shift I students going early to bed early as well as being forced to awake earlier than they desired was significantly higher. Consequently, these Shift-I students achieved a significantly less $(\mathrm{p}=0.0001)$ total sleep duration and $58 \%$ of them were sleep deprived as compared to their counterparts in Shift-II. However, on weekends, majority of students of both the shifts followed a similar pattern of sleep habits of going to bed later than $10 \mathrm{pm}$ and waking later in the morning. Thereby, they comfortably managed to clock total sleep durations of 8 hrs or more. These findings clearly indicate how early school start timings force the students to curb their natural need for sleep leading to decreased sleep durations and increased sleep debt. Due to relaxation of this pressure on weekends, the children tend to "catchup" on their sleep by sleeping late and also getting up late!

Another lesser known, but equally important, factor contributing to this pattern is the delayed sleep phase syndrome (DSPS) normally seen in adolescents. DSPS is a physiological, circadian rhythm variation in which an individual's internal circadian pacemaker is not in synchrony with environmental timings. Affected adolescents typically experience difficulty in initiating and terminating sleep at a "normal" time ${ }^{(16)}$ and prefer to sleep late and wake up late. A shift of about $2 \mathrm{hrs}$ from the normal is seen in these subjects but the sleep structure is otherwise normal.

Surveys addressing sleep habits of older children and adolescents performed in many countries have demonstrated remarkable consistency with regard to developmental changes in sleep habits. With increasing adolescent age, bed timings and waking up time occur later and total sleep time is greater on weekends, suggesting 
that adolescents are attempting to recover sleep during the weekend ${ }^{(16,17)}$. As a consequence of these changes, the total sleep time obtained by adolescents is shorter on school-day compared to weekends as observed in the present study. Carskadon and Wolfson ${ }^{(7)}$ followed 40 students to study the effect of earlier school start time on sleep patterns and observed significant sleep deprivation and daytime sleepiness as the students went to bed later and slept for lesser periods. Significant physiological findings identified were later onset of melatonin secretion, shortened sleep latency, marked increase in incidence of REM (rapid eye movement) sleep and changes in core body ${ }^{(18)}$ temperature. On the same lines, Carskadon's $^{(19)}$ research on the sleep-wake homeostatic process and its interaction with the circadian biological timing system showed evidence of changes during pubertal development which alter sleep patterns substantially. These findings suggest that bio-regulatory mechanisms, controlling sleep in adolescents, delay sleep onset along with early school schedules lead to a significant accumulation of sleep debt. Consequently, this deficit often leads to prolonged sleep periods or catch-up sleep on weekends as the physiological need for sleep created by sleep loss can only be reversed by sleep $^{(20)}$.

\section{Effects of sleep loss}

As observed earlier, the students of Shift I were significantly more sleep deprived, thus reporting a higher incidence of lethargy, napping and sleep difficulties. Chronic sleep deprivation is known to set up a "vicious cycle" of daytime tiredness and napping, which in turn disturbs the normal night sleep pattern further exacerbating the sleep loss. Further, it is evident that daytime sleepiness is a direct effect of insufficient nighttime sleep ${ }^{(21,22)}$. Various animal and human models have also demonstrated that sleep curtailment or fragmented sleep results in excessive daytime sleepiness $^{(23,24)}$.

Due to a combination of delayed sleep phase and forcible early rising, adolescents are not ready to function at their peak potential early in the morning ${ }^{(19)}$. Our study displayed that a majority of children across both shifts found it difficult to get up in the mornings. This finding is supported by The study of Carskadon et al who have suggested that early awakening is harder due to the corresponding change in circadian rhythms ${ }^{(7,19)}$. However, Shift I students were clearly more affected as they reported significantly more episodes of reaching the school later as well as feeling sleepy in the early morning classes. Past studies, have also confirmed that students following the early start timings are sleepier and slower to respond initially after awaking ${ }^{(7)}$

Sleep difficulties, like frequent awakenings at night, and daytime napping were also significantly more in Shift I students. This is in accordance to findings in a previous study ${ }^{(25)}$ which showed that sleep disturbances were more in nappers.

\section{Performance of the students of two shifts}

Students of Shift II had consistently better academic performance, especially in English and Mathematics. A regular and healthy, sleep routine has been demonstrated in several studies ${ }^{(26,27)}$ to have a vital role in good academic performance. In this study, we observed that the students of Shift I were sleep deprived and lacked concentration. Attention lapses and brief moments of inattentiveness have been considered as the main reason for the decrease in cognitive performance during sleep deprivation ${ }^{(28,29)}$. Also, early morning is a difficult time for most students to absorb and remember complicated material. Hansen ${ }^{(4)}$ found in a study of 60 high school students, that they were less alert in early morning classes and had to expend greater effort, though all students performed better in the afternoon. Similarly, Gibson. et.al. ${ }^{(30)}$ showed that significant sleep deprivation and sleepiness among students leads to poor academic achievement. They concluded that sleepiness and sleep deprivation in high school students are common and closely associated with a significant detrimental effect on curricular and extracurricular activities. Sleep phase delay is an important and generally unrecognized factor in sleepiness and needs to be recognized as a normal and frequent feature of adolescence. Wolfson ${ }^{(5)}$ surveyed 3,120 high school students and found that those who reported grades as C,D or F had 25 minutes less weeknight sleep than those who scored A or B grades.

\section{Effect on mood}

Multiple studies have demonstrated coexistence of sleep and mood problems but have failed to pinpoint whether mood changes lead to sleepiness or vice versa. Ryan etal have shown that adolescents with clinical mood disorders (particularly major depressive disorder) reported high rates of sleep disturbances ${ }^{(31)}$. However, others have found that adolescents with sleep problems report increased negative mood and difficulties in mood regulation ${ }^{(32,33)}$. Millman also suggested that sleep deprivation has a negative effect on mood in adolescents and mood changes along with poor school 
performance, in turn lead to greater stress and affective problems ${ }^{(34)}$. The negative affect on mood further interferes with sleep, leading to difficulty in falling asleep and erratic schedules. In the present study, there was no difference in the mood of the subjects of two shifts. The closed knit social and family structure in India ensconces the children to be reared in a protected environment and this may explain the absence of any relationship between sleep deprivation and mood.

\section{Conclusion}

This study clearly demonstrates that early school start timing contribute significantly to sleep deprivation among adolescents, leading to daytime sleepiness and poor academic performance. We hope that the crucial advantage, conferred by late school timings to the student's alertness and performance maybe considered while debating on the ideal school timings for children. However, this study had its own limitations as it was conducted on the students of a single school of a metropolitan city. In modern cities, sleep timings are affected not only due to the physiological shift in bed time (DSPS), but also due to several other social distractions such as television, usage of electronic appliances, computers etc which may have acted as a confounding factor on sleep deprivation and poor academic performance. Further studies, involving students of different socioeconomic group of different cities, are recommended to find the various factors and the extent to which each of them is responsible for the change in bed time, reduced total sleep in adolescents and their effect on mood and academic performance.

\section{ACKNOWLEDGMENTS}

We thank Mr. A.K. Pandey, Principal, Kendriya Vidyalaya, New Delhi and Indian Sleep Disorder Association (ISDA) for their help in this study.

\section{References}

1. Carskadon MA. Patterns of sleep and sleepiness in adolescents. Pediatrician 1990; 17:5-12.

2. Dahl RE, Carskadon MA. Sleep and its disorders in adolescence. In: Ferber R, Kryger $M$, eds. Principles and Practice of Sleep Medicine in the Child. Philadelphia, PA: WB Saunders; 1995:19-27.

3. Carskadon MA, Acebo C. Regulation of sleepiness in adolescents: update, insights, and speculation. Sleep 2002; 25:606-614.

4. Hansen M, Janssen I, Schiff A, Zee P, Dubocovich M: The impact of school daily schedule on adolescent sleep. Pediatrics 2005, 115(6):1555-1561.

5. Wolfson AR, Carskadon MA. Sleep schedules and daytime functioning in adolescents. Child Dev. 1998; 69:875-887.

6. Carskadon MA, Acebo C. Entrainment of sleep and dimlight salivary melatonin onset (DLSMO) in young adolescents using a fixed schedule. Sleep Res. 1997; 26:184

7. Carskadon MA, Wolfson AR, Acebo C, Tzischinsky O, Seifer R. Adolescent sleep patterns, circadian timing, and sleepiness at a transition to early school days. Sleep 1998;21:871-881.

8. Kraemer S, Danker-Hopfe H, Dorn H, Schmidt A, Ehlert I, Herrmann WM. Time-of-day variations of indicators of attention: performance, physiologic parameters, and self assessment of sleepiness. Biol Psychiatry. 2000; 48:1069 $-1080$

9. Mindell JA, Owens JA, Carskadon MA. Developmental features of sleep. Child Adolesc Psychiatr Clin N Am. 1999; 8:695-725

10. Dinges DF, Pack F, Williams K, et al. Cumulative sleepiness, mood disturbance, and psychomotor vigilance performance decrements during a week of sleep restricted to 4-5 hours per night. Sleep. 1997; 20:267

11. Hauri $\mathbf{P}$, LindeS. What kind of an insomniac are you? $\mathbf{I N}$ : No More Sleepless nights. John Wiley \& Sons Inc. New York $1996 ; 23-42$

12. Johns MW. A new method for measuring daytime sleepiness: the Epworth sleepiness scale. Sleep 1991;14(6): 540-545.

13. Carskadon MA, Harvey K, Duke P, Anders TF, Litt IF, Dement WC. Pubertal changes in daytime sleepiness. Sleep 1980; 2:453-460

14. Labyak SE, Acebo C, Seifer R, Carskadon MA. Adolescent sleep: preliminary report of a week of actigraphy. Sleep 1999; 22 (Suppl 1):242.

15. Carskadon MA. The second decade. In: Guilleminault C, editor. Sleeping and waking disorders: Indications and Techniques. Menlo Park, CA: Addison Wesley; 1982. p. 99-125.

16. Weitzman ED, Czeisler CA, Coleman RM, et al. Delayed sleep phase syndrome. Achronobiological disorder with sleeponset insomnia. Arch Gen Psychiatry. 1981; 38:737746.

17. Pelayo RP, Thorpy MJ, Glovinsky P. Prevalence of delayed sleep phase syndrome among adolescents. Sleep Res. 1998; 17:391.

18. Shibui K, Uchiyama M, Okawa M. Melatonin rhythms in delayed sleep phase syndrome. J Biol Rhythms. $1999 ; 14: 72-76$

19. Carskadon MA, Acebo C, Jenni OG: Regulation of adolescent sleep: implications for behavior. Ann NY Acad Sci 2004, 1021:276-91.

20. Rogers NL, Dorrian J, Dinges DF: Sleep, waking and neurobehavioural performance. Frontiers in Bioscience 8, s1056-1067, (2003).

Indian Journal of Sleep Medicine (IJSM), Vol. 5, No. 3, 2010 
21. Roehrs T, Zorick F, Sicklesteel J, Witting R, Roth T. Excessive daytime sleepiness associated with insufficient sleep. Sleep $1983 ; 6: 319-325$.

22. Strauch I, Meier B. Sleep need in adolescents: A longitudinal approach. Sleep 1988; 11:378-86.

23. Leibowitz SM, Lopes MC, Andersen ML, Kushida CA. Sleep deprivation and sleepiness caused by sleep loss. Sleep Med Clin 2006;(1):31-45

24. Suri JC, Sen MK, singh P, Kumar R, Aggarwal P. Sleep patterns and their impact on lifestyle, anxiety and depression in BPO workers. Indian J sleep Med 2007; 2.2, 64-70.

25. Singh $\mathbf{R}$, Sharma R, Suri JC, Das S.Is daytime napping a healthy habit in adolescents? Indian J Sleep Med 2009; $4.4,136-142$.

26. Taub J, Berger R (1973). Performance and mood following variations in the length and timing of sleep. Psychobiology, $10(6), 559-570$.

27. Singh R, Sharma R, Suri JC, Das S. Impact of sleep patterns on mood and academic performance of medical students. Indian J Sleep Med 2009; 4.2, 61-67.
28. Dorrian J, Rogers NL, Dinges DF. Psychomotor vigilance performance: Neurocognitive assay sensitive to sleep loss. New York: Marcel Dekker. 2005; p. 39-70.

29. Kjellberg A. Sleep deprivation and some aspects of performance. II. lapses and other attentional effects. Waking Sleeping, 1977; 1:145-8.

30. Gibson ES, Powles AC, Thabane L, et al. "Sleepiness" is serious in adolescence: two surveys of 3235 Canadian students. BMC Public Health 2006; 6:116.

31. Ryan ND, Puig-Antich J, Ambrosini P, et al. The clinical picture of major depression in children and adolescents. Arch Gen Psychiatry. 1987; 44:854-861.

32. Morrison DN, McGee R, Stanton WR. Sleep problems in adolescence. J Am Acad Child dolesc Psychiatry. 1992; 31:94-99.

33. Price VA, Coates TJ, Thoresen CE, Grinstead OA. Prevalence and correlates of poor sleep among adolescents. Am J Dis Child. 1978; 132:583-586.

34. Millman RP. Excessive Sleepiness in Adolescents and Young Adults: Causes, Consequences, and Treatment Strategies. Pediatrics 2005; 115:1774-1786. 